\title{
Kann und darf das Handy gegen Corona helfen?
}

\section{Pressemitteilung des Landesbeauftragten für den Datenschutz und die Informationsfreiheit Rheinland-Pfalz vom 31.03.2020}

Angesichts der weiteren Ausbreitung des Corona-Virus werden derzeit an vielen Stellen Tracking-Lösungen zur Bekämpfung der Corona-Pandemie diskutiert. Der Bundesgesetzgeber hat zwar aufgrund der Bedenken der Datenschutzbeauftragten und des Justizministeriums zunächst davon Abstand genommen, für die Verwendung von Mobilfunk-Standortdaten für Zwecke des Infektionsschutzes eine eigene Rechtsgrundlage zu schaffen, die Idee wird angesichts der vermuteten Dauer der Pandemie voraussichtlich jedoch wieder aufgegriffen werden.

Die Frage ist dabei, welchen technischen Weg man wählt. Auswertungen auf Basis einer Funkzellenabfrage sind ungeeignet https://gdprhub.eu/index.php?title=Projects_using_personal_ data_to_combat_SARS-CoV-2, weil die Zellen in den meisten Fällen zu groß und damit für die Identifizierung von Kontakten der Mobilfunkteilnehmer untereinander ungeeignet sind.

Der in einigen anderen Staaten beschrittene Weg, im Rahmen eines Überwachungspakets Standortdaten mit anderen Daten zusammenzuführen (Kreditkarte, Videoüberwachung usw.) erscheint vor dem Hintergrund des europäischen Datenschutzverständnisses unverhältnismäßig.

Dies ist aber auch gar nicht notwendig. Um Infektionsketten nachvollziehen zu können, reichen reine Standortdaten alleine ohnehin nicht aus; ausschlaggebend ist die Kenntnis von Kontakten mit infizierten Personen. Hier scheint eher der Weg über eine Handy-App gangbar, die auf der Grundlage einer freiwilligen Nutzung bei relevanten Kontakten die Betroffenen informiert.
Derartige Lösungen werden gegenwärtig an verschiedenen Stellen und mit teils unterschiedlichen Ansätzen verfolgt.

„Vieles hängt hier an der konkreten Ausgestaltung“, so der Landesbeauftragte für den Datenschutz und die Informationsfreiheit, Prof. Dr. Dieter Kugelmann, „aber eine solche App ist möglich und auch datenschutzkonform gestaltbar. Dazu machen sich gerade viele Leute Gedanken und ich hoffe, dass man dabei die Bedeutung unserer Freiheitsrechte nicht aus den Augen verliert. Für mich sind dabei folgende Kriterien entscheidend: die Freiwilligkeit zur Entscheidung über die Nutzung, eine enge Zweckbindung, die Pseudonymisierung der Daten sowie eine Löschung, wenn eine Infektionsgefahr nicht mehr gegeben ist. Für mich ist der Kern der Sache, dass wir nicht vorschnell Freiheit aufgeben, wenn es dem effektiven Gesundheitsschutz gar nicht dient. Es besteht kein Zweifel, dass die Bekämpfung der Pandemie Priorität genießt, aber bitte im rechtsstaatlichen Rahmen, sonst erodiert die staatliche Ordnung“.

Weitere Informationen:

- Handy-Tracking vs. Corona https://www.datenschutz.rlp.de/fileadmin/lfdi/Dokumente/ Handy-Tracking_vs._Corona.pdf

- Paper „Corona-Tracking“ des European Centers for Digital Rights

https://noyb.eu/sites/default/files/2020-03/ad_hoc_paper_ corona_tracking_v0.2_2.pdf

- Übersicht „Projects using personal data to combat SARS-CoV-2“ https://gdprhub.eu/index.php?title=Projects_using_personal_ data_to_combat_SARS-CoV-2

Prof. Dr. Dieter Kugelmann, Der Landesbeauftragte für den Datenschutz und die Informationsfreiheit Rheinland-Pfalz

\section{Springer}

\section{Neues aus der Reihe „Die blaue Stunde der Informatik“}

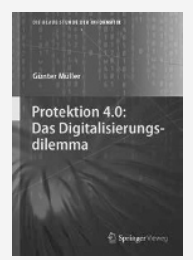

G. Müller

Protektion 4.0: Das Digitalisierungsdilemma

Reihe: Die blaue Stunde der Informatik

2020, XI, 241 S. 34 Abb. Geb.

$€(D) 49,99 \mid €$ (A) 51,39 |*CHF 55.50 | ISBN 978-3-662-56261-1

$€ 39,99 \mid$ *CHF 44.00 | ISBN 978-3-662-56262-8 (eBook)

\author{
Ihre Vorteile in unserem Online Shop: \\ Über 280.000 Titel aus allen Fachgebieten | eBooks sind \\ auf allen Endgeräten nutzbar | Kostenloser Versand für \\ Printbücher weltweit
}

$€(D)$ : gebundener Ladenpreis in Deutschland, $€$ (A): in Österreich *: unverbindliche Preisempfehlung. Alle Preise inkl. MwSt. 